\title{
Experimental studies of strong dipolar interparticle interaction in monodisperse $\mathrm{Fe}_{3} \mathrm{O}_{4}$ nanoparticles
}

\author{
Che Jin Bae, S. Angappane, and J.-G. Park ${ }^{\mathrm{a})}$ \\ BK21 Physics Division, Department of Physics, SungKyunKwan University, Suwon 440-746, Korea \\ Youjin Lee, Jinwoo Lee, Kwangjin An, and Taeghwan Hyeon \\ National Creative Research Initiative Center for Oxide Nanocrystalline Materials and School of Chemical \\ Engineering, Seoul National University, Seoul 151-744, Korea
}

(Received 18 March 2007; accepted 10 August 2007; published online 4 September 2007)

\begin{abstract}
Interparticle interaction of monodisperse $\mathrm{Fe}_{3} \mathrm{O}_{4}$ nanoparticles has been experimentally investigated by dispersing the nanoparticles in solvents. With increasing the interparticle distances to larger than $100 \mathrm{~nm}$ in a controlled manner, the authors found that the blocking temperature $\left(T_{B}\right)$ of the nanoparticles drops continuously and eventually gets saturated with a total drop in $T_{B}$ of 7-17 K observed for 3, 5, and $7 \mathrm{~nm}$ samples, compared with their respective nanopowder samples. By carefully studying the dependence of $T_{B}$ on the interparticle distance, the authors could demonstrate that the experimental dependence of $T_{B}$ follows the theoretical curve of the dipole-dipole interaction. (C) 2007 American Institute of Physics. [DOI: 10.1063/1.2778758]
\end{abstract}

The past few years have seen the field of nanoparticles virtually explode and nowadays they find possible applications in very diverse areas such as high-density magnetic storage devices and biomedicine, to name only a few. ${ }^{1,2}$ In particular, that one can produce nanoparticles with a very narrow size distribution, or the so-called monodisperse, and in high reproducibility ${ }^{3-6}$ rendered it possible to focus on their more intrinsic properties and associated applications. ${ }^{7,8}$ In most prospective applications, nanoparticles are used as in solutions or nanopowders. Therefore, deeper understanding of the magnetic properties of individual nanoparticles in solution and their mutual interactions will be invaluable for numerous potential applications as well as for fundamental scientific interests.

In the early 1990s, interparticle interaction was studied as origin of rather unusual glassy behavior found in nanoparticles. ${ }^{9}$ Nanoparticles diluted using paraffin were also recently shown to have blocking temperature enhanced with increasing concentration of nanoparticles, which is attributed to their strong dipolar interaction. ${ }^{10}$ In spite of this seemingly immediate importance of the interparticle interaction, however, there has been less systematic experimental studies on the nature of the interparticle interaction. In this letter, we present extensive studies of the magnetic properties of monodisperse $\mathrm{Fe}_{3} \mathrm{O}_{4}$ nanoparticles dispersed in different solvents, and convincingly show that the primary interaction between nanoparticles is, as expected, the dipole-dipole interaction.

For our studies, we have prepared uniform and highly crystalline nanoparticles of $\mathrm{Fe}_{3} \mathrm{O}_{4}$ by reverse micelles method, employing microemulsions as nanoreactors and hydrated iron salts as reactants. ${ }^{11}$ As shown in Fig. 1, transmission electron microscopy (TEM) images of the 3, 5, and $7 \mathrm{~nm}$ particles attest that our nanoparticles are uniform in shape and in size: $3 \pm 0.34,5 \pm 0.37$, and $7 \pm 0.37 \mathrm{~nm}$. Another noteworthy point is that our high resolution TEM (HRTEM) images shown in the insets demonstrate the good crystalline

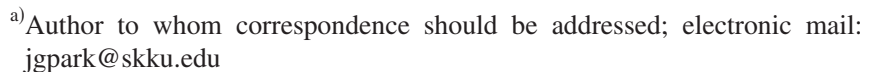

nature of the particles. Therefore, we shall ignore any possible effects related to the size distribution of the nanoparticles in the following discussion of our results. We note that once we washed out carefully the outmost layers of surfactants from the nanoparticles, the resulting powders could easily be redispersed in solvent without sign of segregation in our HRTEM measurements. For our studies, we used two solvents: oleic acid for less than $15 \mathrm{~nm}$ of interparticle distance and $p$-xylene for more than $15 \mathrm{~nm}$.

dc magnetization measurements were made using a superconducting quantum interference device magnetometer in an applied field of 100 Oe. We limited our measurements to a relatively low temperature range from 5 to $180 \mathrm{~K}$ for the solution samples to ensure that the nanoparticles are completely frozen in ferrofluid during the entire measurement. Figure 2 shows the magnetization curves of 3,5, and $7 \mathrm{~nm}$ $\mathrm{Fe}_{3} \mathrm{O}_{4}$ particles measured before and after being fully dispersed in solution. The zero-field-cooled (ZFC) magnetization $\left(M_{\mathrm{ZFC}}\right)$ of all the nanoparticles shows the typical peak features at certain temperatures, the so-called blocking temperature $T_{B}$, and decreases thereafter. On the other hand, the field-cooled $(\mathrm{FC})$ magnetization $\left(M_{\mathrm{FC}}\right)$ exhibits similar behavior to $M_{\mathrm{ZFC}}$ above $T_{B}$ and then becomes leveled off at lower temperatures. As shown in Fig. 2, the blocking temperatures of the nanoparticles diluted in the solution are different from those of the nanopowder samples. For example, the blocking temperatures are 28,66 , and $98 \mathrm{~K}$ for 3,5 , and $7 \mathrm{~nm}$ particles in solution, whereas the corresponding nanopowders have $T_{B}$ of 45,75 , and $105 \mathrm{~K}$, respectively. Therefore, when fully dispersed in $p$-xylene, $T_{B}$ decreases by about 17,9 , and $7 \mathrm{~K}$ for 3,5 , and $7 \mathrm{~nm}$, respectively. This decrease in $T_{B}$ indicates that the interparticle interaction is weakened in the solution, and similar drops of $T_{B}$ were previously reported with increasing interparticle distance too. ${ }^{12,13}$ Thus, the interparticle interaction of the $\mathrm{Fe}_{3} \mathrm{O}_{4}$ nanoparticles is found to play a significant role in the magnetic properties of these nanoparticles.

Dipole-dipole interaction energy among the nanoparticles can be written as follows: ${ }^{14}$ 


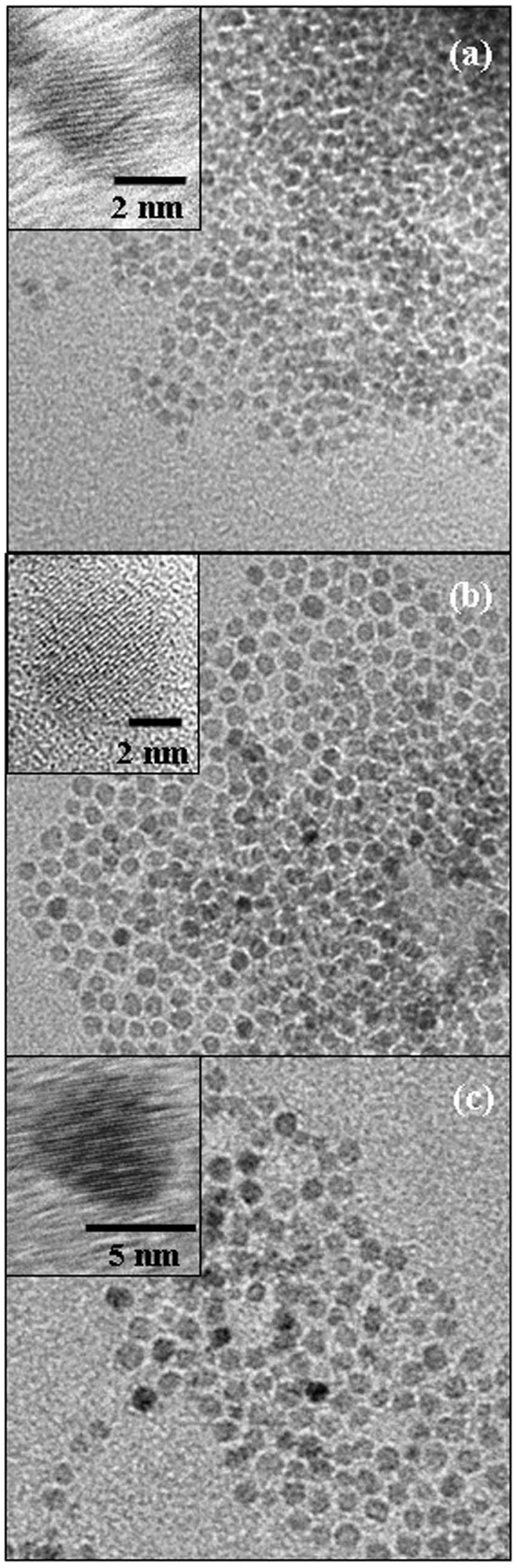

FIG. 1. TEM images of $\mathrm{Fe}_{3} \mathrm{O}_{4}$ nanoparticles with three particle sizes (a) $3 \pm 0.34$, (b) $5 \pm 0.37$, and (c) $7 \pm 0.37 \mathrm{~nm}$ (from top to bottom). The insets are high-resolution TEM images showing the good crystalline nature of the nanoparticles.

$$
E_{\mathrm{int}}=\frac{\mu_{0}}{4 \pi r^{3}}\left[\boldsymbol{\mu}_{\mathbf{1}} \cdot \boldsymbol{\mu}_{2}-\frac{3}{r^{2}}\left(\boldsymbol{\mu}_{\mathbf{1}} \cdot \mathbf{r}\right)\left(\boldsymbol{\mu}_{\mathbf{2}} \cdot \mathbf{r}\right)\right],
$$

where $\mu_{1}$ and $\mu_{2}$ are magnetic moments of neighboring particles with interparticle distance $r$. For samples with ranDownloaded 06 Sep 2007 to 147.46 .143 .240 . Redistribution subject

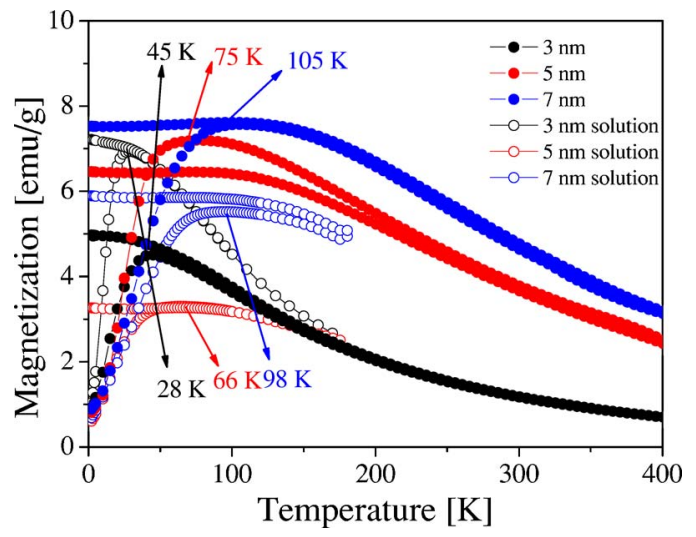

FIG. 2. (Color online) Zero-field-cooled (ZFC) and field-cooled (FC) magnetizations of the $\mathrm{Fe}_{3} \mathrm{O}_{4}$ nanoparticles before and after being fully dispersed in solution. The interparticle distances in the solution are estimated to be about $60-120 \mathrm{~nm}$.

domly distributed interacting nanoparticles in solution, Eq. (1) should be averaged over for all possible isotropic orientations of two neighboring nanoparticles and the resulting expression for the interaction energy then becomes,

$$
E_{\mathrm{int}}=\frac{\mu_{0} \mu^{2}}{4 \pi r^{3}}\left(\frac{\pi}{4}\right)^{2}
$$

where $\mu$ is now an average moment of the particles. When the nanoparticles are separated by about $7 \mathrm{~nm}$ away from one another, the energy of the magnetic dipolar interaction calculated using Eq. (2) is estimated to be $0.33 \mathrm{meV}$, corresponding to $3.8 \mathrm{~K}$. This decrease in the theoretical interparticle interaction is in good agreement with our experimental results with a full drop in $T_{B}$ of $9 \mathrm{~K}$ for the $5 \mathrm{~nm}$ particles. This demonstrates that a main interaction term of the nanoparticles is predominantly dipole-dipole interaction. To further explore this point, we made systematic variations of the interparticle distance for the 5 and $7 \mathrm{~nm} \mathrm{Fe}_{3} \mathrm{O}_{4}$ nanoparticles and studied their magnetic properties.

We have dispersed the nanoparticles in solvents followed by $1 \mathrm{~h}$ sonication each time. Using this method, we could control the interparticle distance up to $130 \mathrm{~nm}$ reasonably well. Figure 3 is the summary of our results, showing how the blocking temperature changes with the interparticle distance. Initially, the blocking temperatures drop rather quickly with small changes in the interparticle distance and then flatten out at a certain value of $T_{B}$. In order to establish the origin of the observed drop in $T_{B}$, we have then fitted the results against the following theoretical formula based on a simple dipole-dipole interaction model,

$$
T_{B}=T_{0}+\frac{C}{r^{n}},
$$

where $T_{0}$ is a blocking temperature of isolated nanoparticles and $C$ is a constant of the magnetic dipolar interaction strength. The solid lines of Fig. 3 are for the fitting results using Eq. (3) with $n=3$, i.e., the typical dipole-dipole interaction. As one can see, the theoretical curves follow the experimental results reasonably well. However, we also note that for the $5 \mathrm{~nm}$ particles some discrepancy exists below $40 \mathrm{~nm}$ between the theoretical curve (No. 1) and the data, which, we found, could be improved if we used a log-normal distribution function for the interparticle distance (dashed line No. 2). We believe that this inverse-cube dependence of
to AIP license or copyright, see http://apl.aip.org/apl/copyright.jsp 


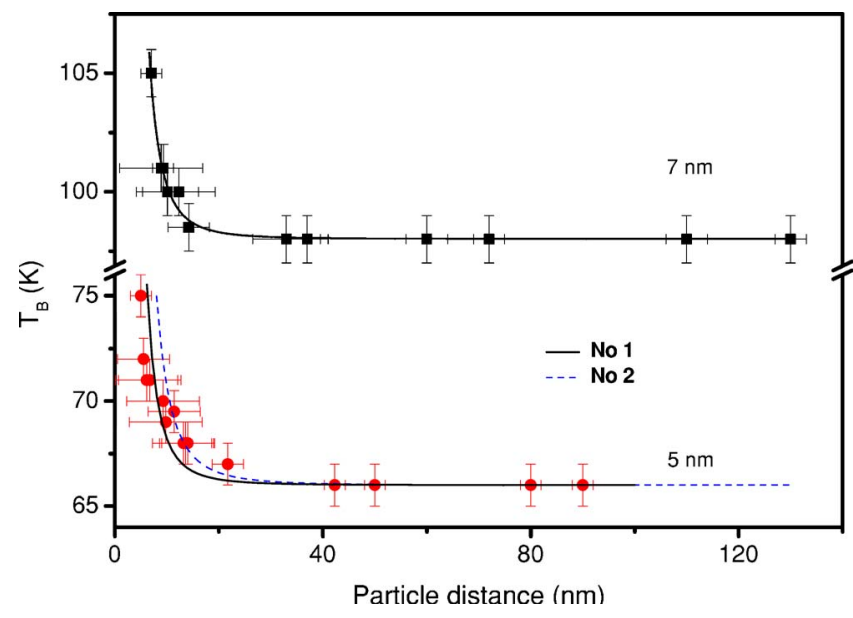

FIG. 3. (Color online) Variation of the blocking temperatures with the interparticle distance for $7 \mathrm{~nm}$ (top) and $5 \mathrm{~nm}$ (bottom) $\mathrm{Fe}_{3} \mathrm{O}_{4}$ nanoparticles in solution. The solid lines (No. 1) are fit to a simple dipole-dipole interaction model, while the dashed line (No. 2) for the $5 \mathrm{~nm}$ particles data is produced using a formula for the dipole-dipole interaction model with a log-normal distribution of interparticle distance. The first data point in each figure is for the undiluted powder samples.

the blocking temperature on the interparticle distance proves convincingly that the interparticle interaction among the $\mathrm{Fe}_{3} \mathrm{O}_{4}$ nanoparticles is dominated by the dipole-dipole-type interaction. We have also confirmed basically the same behavior using another monodisperse $6 \mathrm{~nm} \mathrm{Fe}_{3} \mathrm{O}_{4}$ nanoparticles prepared by a "heating-up" method (not shown here). ${ }^{3}$ Hence, the dipolar interaction among the nanoparticles plays a key role in the magnetic properties of the nanoparticles in solution. Closely related to our work, we found that the theoretical simulations by Kechrakos and Trohidou ${ }^{15}$ have shown that the blocking temperature of the arrays of Co nanoparticles varies with the inverse cube of the interparticle spacing $\left(T_{B} \sim d^{-3}\right)$, which indicates that the additional energy barrier for magnetization reversal is proportional to the dipolar coupling strength. Unfortunately, however, no systematic experimental studies have been reported yet for the monodisperse nanoparticle systems. With our work and, in particular, the method of accurately controlling the interparticle distance of $\mathrm{Fe}_{3} \mathrm{O}_{4}$ nanoparticles, it will be highly interesting and important to extend our studies to other nanoparticles. Furthermore, it would be desirable to study the spin dynamics of the nanoparticles while controlling the interparticle dis- tances, which is an important issue in ferrofluids.

To summarize, we have investigated the contribution of the dipole-dipole interaction to the blocking behavior of the magnetic nanoparticles using monodisperse $\mathrm{Fe}_{3} \mathrm{O}_{4}$. With a simple theoretical model of dipolar interaction, we could explain the observed behavior reasonably well. Our study demonstrates the key role of the dipolar interaction in better understanding the magnetic properties and, more importantly, the spin dynamics of the nanoparticles; the knowledge of which will be vital for future applications of magnetic nanoparticles in various industrial applications.

Work at SungKyunKwan University was supported by the BK21 program, the CSCMR, the Korea Research Foundation (Grant No. 2005-C00153), and the BAERI program. One of the authors (J.G.P) acknowledges the LG Yonam Foundation for supports, and KEK for hospitality, where the final draft was prepared. Another author (T.H.) would like to thank the financial support from the National Creative Research Initiative Program.

${ }^{1}$ G. Reiss and A. Hütten, Nat. Mater. 4, 725 (2005).

${ }^{2}$ Scientific and Clinical Applications of Magnetic Carriers, edited by U. Hafeli, W. Schutt, J. Teller, and M. Zborowski (Plenum, New York, 1997), p. 569.

${ }^{3}$ J. Park, K. An, Y. Hwang, J.-G. Park, H. J. Noh, J. Y. Kim, J. H. Park, N. M. Hwang, and T. Hyeon, Nat. Mater. 3, 891 (2004).

${ }^{4}$ J. Park, E. Lee, N. M. Hwang, M. Kang, S. C. Kim, Y. Hwang, J.-G. Park, H. J. Noh, J. Y. Kim, J. H. Park, and T. Hyeon, Angew. Chem., Int. Ed. 44, 2872 (2005).

${ }^{5}$ Young Soo Kang, Subhash Risbud, John F. Rabolt, and Pieter Stroeve, Chem. Mater. 8, 2209 (1996).

${ }^{6}$ T. Fried, G. Shemer, and G. Markovich, Adv. Mater. (Weinheim, Ger.) 13, 1158 (2001).

${ }^{7}$ M. Todorovic, S. Schultz, J. Wong, and A. Scherer, Appl. Phys. Lett. 74, 2516 (1999).

${ }^{8}$ T. Reese, B. Bjelke, R. Porszasz, D. Baumann, D. Bochelen, A. Sauter, and M. Rudin, NMR Biomed. 13, 43 (2000).

${ }^{9}$ W. Luo, S. R. Nagel, T. F. Rosenbaum, and R. E. Rosensweig, Phys. Rev. Lett. 67, 2721 (1991).

${ }^{10}$ J. M. Vargas, W. C. Nunes, L. M. Socolovsky, M. Knobel, and D. Zanchet, Phys. Rev. B 72, 184428 (2005).

${ }^{11}$ Y. Lee, J. Lee, C. J. Bae, J.-G. Park, H. J. Noh, J. H. Park, and T. Hyeon, Adv. Funct. Mater. 15, 503 (2005).

${ }^{12}$ J. M. Vargas, W. C. Nunes, L. M. Socolovsky, M. Knobel, and D. Zanchet, Phys. Rev. B 72, 184428 (2005).

${ }^{13}$ M. F. Hansen and S. Mørup, J. Magn. Magn. Mater. 184, 262 (1998).

${ }^{14}$ David J. Griffiths, Introduction to Electrodynamics, 3rd ed. (Prentice-Hall, New York, 1999), p. 165.

${ }^{15}$ D. Kechrakos and K. N. Trohidou, Appl. Phys. Lett. 81, 4574 (2002). 